Jpn. J. Genet. (1989) 64, pp. 295-313

\title{
REVIEW
}

\section{Composition of eukaryotic gene loci regarding gene conversion units and the presence or the absence of intralocus reciprocal recombination}

\author{
Yoshiaki KITANI \\ Takasago Research Institute, Kamata, Tokyo 144
}

(Received 13 February 1989)

\begin{abstract}
-In Sordaria fimicola, the $g$-locus is composed of a single convertron and does not show intralocus reciprocal recombination. On the contrary, the $i$-locus is composed of multiple convertrons and shows genuine intralocus reciprocal recombination.-
\end{abstract}

\section{Introduction}

Recent reports on the separate occurrence of reciprocal recombination from gene conversion events (Roman and Fabre, 1983; Klein, 1984; Willis and Klein, 1987; Bollag and Liskey, 1988) support the previous finding by Kitani (1982), and provide a favorable atomosphere of expressing the fact. The fact is that there are two kinds of loci in Sordaria fimicola; one in which reciprocal recombination does not occur, and the other in which it occurs.

The only locus that has so far been known to show a uniform frequency of gene conversion, throughout one-point crosses of all the alleles in the locus and all possible interellelic crosses (Kitani and Olive, 1967, 1969, 1970; Kitani and Whitehouse, 1974), is the grey ascospore locus (g-locus) of $S$. fimicola. At the same time, the $g$-locus has not shown any sign of the occurrence of genuine intralocus reciprocal recombination. On the contrary, the indigo ascospore locus ( $i$-locus) of $S$. fimicola was, however, appeared to undergo intralocus reciprocal recombination, and showed various conversion frequencies in interallelic crosses (Kitani, 1982).

Various data from the $g$-locus analysis suggest that gene conversion and conversion-associated flanking marker exchanges are the phenomena arising from an integrated mechanism (Kitani and Olive, 1967, 1969, 1970, Kitani, 1978a, $1978 \mathrm{~b}, 1982)$. And the data from the $i$-locus analysis suggest that intralocus reciprocal recombination occurs by a separate mechanism from the one for gene conversion and its associated flanking marker exchanges (Kitani, 1982). Thus, 
having two types of locus in a single organism, one with and the other without the ability of intralocus reciprocal recombination, one may expect to determine the DNA structure (base sequence) responsible for the reciprocal recombination, if the complete nucleotide sequences for the loci $g$ and $i$ are determined and compared with each other.

Although the characteristics of the $i$-locus in conversion and recombination were previously published (Kitani, 1982) stating the above explained differences between the loci $g$ and $i$, significance of the explained facts are not yet understood well by the recombination researches working on other organisms. The difficulty like this of the Sordaria work to acquire general understandings is due primarily to the entire lack of a gene locus in other organisms similar to the $g$-locus in characteristics. Therefore, a brief review for exposing the critical differences between the two loci, $g$ and $i$, is presented in Section II of this article.

Among the conversion ascus types, 6 in one-point crosses and 21 in interallelic crosses (Kitani and Olive, 1967, 1969), some are indistinguishable from the normal background asci; these ascus types are normal 4:4 conversion type in one-point crosses and $\mathrm{E}$ type in interallelic crosses (at all the times), and most of aberrant 4:4 asci in one-point crosses and $F$ type in interallelic crosses when an unlinked colour marker is absent. Crosses for the $g$-locus have been equipped with the unlinked colour marker, indigo, but the crosses for the $i$-locus were not. Although such distortions of the recorded conversion frequency from the real is insignificant for general porposes, especially in interallelic crosses, it could be serious when one-point cross conversion frequency is the critical element in an analysis. Therefore, a critical reexamination was carried out using corrected one-point cross conversion frequencies on the previously reported interallelic cross conversion frequencies in the $i$-locus (kitani, 1982). The results of this reexamination are presented in Section III of this article.

In eukaryotes, comprehensive analysis of meiotic recombination events can ideally be done through meiotic octads, instead of tetrads, because meiotic bivalents are composed of four DNA double helices representing eight potential genic units, and the actual presence of postmeiotic segregation in gene conversion spectra. But, as the requirments are so severe and unmerous for the ideal systems of octad analysis, no organism has so far acquired a gene locus with a perfect octad analysis system. The system closest to fulfil the requirments, and has provided substantial data, is the $g$-locus system of $S$. fimicola. And the lower limit of frequencies analysed in statistically reliable sizes is the order of $10^{-3}$ for comprehensive recombination phenomena. As a matter of fact, eukaryotic interlocus crossing over, gene conversion, and conversion-associated flanking marker exchanges are all at or above the frequency order of $10^{-3}$.

While in the materials of no octad formation, or in the analysis of mitotic recombination events at molecular level, etc., random spore or random product analysis has been applied. This method produced a large amount of data on 
molecular details of recombination events at the far lower frequencies than octad analysis can handle. Based on these mostly molecular information and the concepts developed with prokaryotic organisms, recombination theories such as those by Meselson and Radding (1975), and by Fogel et al. (1979), were developed, and are so far enjoying overwhelming acceptance. However, elucidation to bridge the events of $10^{-3}$ frequency order obtained through critical octad analysis and the theories based on the events of lower frequencies is left for the future. Therefore, a proposed mechanism for the recombination events in eukaryotes at the level of octad analysis and common through meiosis and mitosis is given in Section IV of this article, based both on the previous explanations (Kitani and Olive, 1969; Kitani, 1979, 1982) and the present analysis.

\section{Composition and characteristics of the loci $g$ and $i$ regarding the absence of intralocus reciprocal recombination}

\section{1) Conversion frequencies in crosses of the g-colus mutants}

All the tested $g$-locus alleles showed virtually the same total conversion frequency in both one-point and interallelic crossies (Kitani and Olive, 1967, 1969, 1970; Kitani and Whitehouse, 1974; Kitani, 1962, 1978, 1988). This constant conversion frequency could have resulted if all 12 mutant alleles, which have different colour expressions, were tightly clustered within the $g$-locus. But this seems unlikely. A more likely hypothesis is that the locus functions as a single unit with respect to conversion. In other loci where conversion frequencies in interallelic crosses are the same or exceed the expectation from adding frequencies for one-point crosses, it is suggested that the two mutant sites are located in different parts of the multi-convertron-locus. This is consistent with the $i$-locus conversion (Kitani, 1982), which will be discussed later.

The conversion frequency at the $g$-locus was not modified even under conditions where conversion spectra were modified (Kitani and Olive, 1970; Kitani and Whitehouse, 1974). The uniformity in conversion frequencies suggests the absence of reciprocal recombination within the $g$-colus.

\section{2) Recognition of intralocus reciprocal recombination}

In one-point crosses, intralocus reciprocal recombination cannot be recognised, irrespective of the origin, apparent or genuine. However, the frequency of apparent reciprocal recombination can be estimated as class $R p$ (flanking marker recombination present) of normal 4:4 type conversion asci through a method given in Table 3 and Fig. 2 of Kitani and Olive (1967). In interallelic crosses, however, intralocus reciprocal recombination, regardless whether it is apparent or genuine, can be obtained as a part of the $M$ (or e) type ascus population shown in Figs. 3 and 4 of Kitani and Olive (1969). This gene conversion ascus type is phenotypically indistinguishable from tetratype asci. Should the total conversion frequen- 
cies be the same between both for one point and for interallelic crosses, there would be no room for genuine reciprocal recombination in the population of $M$ type asci. On the other hand, should the total conversion frequencies be higher in interallelic crosses showing a large proportion for $M$ type, the increased portion of the total conversion frequency would be due to the presence of genuine reciprocal recombinant asci.

The $g$-locus appeared to have no intralocus reciprocal recombination by these criteria (Kitani and Oliver, 1967, 1969; kitani, 1978).

\section{3) Flanking marker exchanges}

Flanking marker exchanges can be recorded in conversion asci of 6:2 and 5:3 ratios and aberrant 4:4 asci (see Table 8 of Kitani and Olive, 1967) in one-point crosses. However, occurrence of double orossing over flanking both sides of the $g$-locus can be detected only in asci showing 5:3 ratio (Kitani and Olive, 1967). Although this marker exchange subclass (Ra-2) appears in the phenotypical recombination class $\mathrm{Ra}$ (flanking marker recombination absent), the presence of Ra-2 should not be ignored, even though it has been inevitably overlocked in yeast due to the lack of octad asci, and therefore ignored in the implications; whether this subclass is taken into account or not makes a decisive difference for theories of the mechanism of recombination. The proportion of classes $\mathrm{Ra}$ and $\mathrm{Rp}$ (recombination present) is roughly 1:1 in one-point crosses (Kitani and Olive, 1967).

Flanking marker exchanges can be recorded even in conversion asci that show normal 4:4 segregation in the $g$-locus interallelic crosses (Kitani and Olive, 1969; Kitani, 1978). Although the 1:1 ratio is roughly kept in the $g$-locus interallelic crosses, factors affecting this ratio are numerous. Among these factors, differential preference in the mode of base correction between the chromatids involved in the conversion event is serious between subclasses of some individual asscus types (Kitani and Olive, 1969). And this kind of distortion might be closely related with the phenomenon of conversion spectrum alteration (Kitani aced Olive, 1970; Kitani and Whitehouse, 1974).

Another factor which would theoretically increase the proportion of Rp over Ra is the presence of genuine intralocus reciprocal recombination; the frequency of this event is correlated with the increased proportion of class Rp. Conversely, maintenance of the 1:1 ratio for $R a: R p$ in interallelic crosses would be a good indication of the absence of genuine intralocus reciprocal recombination in this particular locus, the $g$-locus.

Flanking marker exchange associated with gene conversion is, therefore, different from intralocus reciprocal recombination, even though the result is phenotypically the same. This is therefore, signified as "conversion-associated apparent reciprocal recombination", and by iteself, an integrated part of gene conversion event. 


\section{4) The absence of interference in association with the g-locus gene conversion}

The presence of interference between exchanges of markers near the $g$-locus has been known since 1963 (Perkins et al., 1963). This information came from asci in which the $g$-locus was not involved in a conversion event and therefore showed a normal 4:4 ratio. However, when the $g$-locus is involved in a conversion event, interference is absent both in one-point and interallelic crosses (Kitani, 1978). Should genuine intralocus recombinations occur in association with the $g$-locus conversion, these recombinations would interfer with the normal exchanges of flanking markers. Therefore, the absence of interference in association with the $g$-locus gene conversion is the powerful evidence for the lack of intralocus reciprocal recombination within the $g$-locus. This result also suggests that gene conversion (with conversion-associated fianking marker exchanges) and genuine reciprocal crossing over (regardless of intra- or inter-locus event) do not share a single mechanism.

This concept is at least partially in agreement with the recent findings in Saccharomyces (Roman and Fabre, 1983; Klein, 1984; Willis and Klein, 1987) and mammalian cells (Bollag and Liskay, 1988).

\section{5) Multiple unit structure of the i-locus}

Conversion frequencies in one point crosses of five indigo alleles and five blue alleles of the $i$-locus appeared nearly uniform around $10 \times 10^{-3}$, when not associated with a dominant $\mathrm{Rec}^{+}$factor (Kitani, 1982). This tendency is similar to that of the $g$-locus (Kitani and Olive, 1967; Kitani, 1982). For the $i$-locus, however, the need to correct the observed frequencies (Kitani and Olive, 1967) is more serious than for the $g$-locus, because ovarlooked ascus types include both aberrant and normal 4:4 types for the $i$-locus (Kitani, 1982), but only normal 4:4 type for the $g$-locus (Kitani and Olive, 1967).

In interallelic crosses, however, the results in conversion frequencies appeared remarkably different between the loci $g$ and $i$ (Kitani, 1982; Kitani and Olive, 1969; Kitani, 1978). Based on the uncorrected one-point cross conversion frequencies, interallelic conversion frequencies of all 25 combinations in the $i$-locus appeared to be at least double the one-point cross conversion frequencies (Kitani, 1982), with or without a variety of additional values (Kitani, 1982). The difference in interallelic conversion frequencies between the loci $g$ and $i$ means that the $g$-locus is composed of a single "Holliday-Whitehouse" conversion unit (Holliday, 1964 and Whitehouse, 1963), and the $i$-locus is composed of at least two "homoeoconversion-units".

\section{6) The presence of reciprocal recombination as $M$ type asci in the $i$-locus}

In interallelic crosses, 21 conversion ascus phenotypes are determined by the combination of six fundamental ascus types for the two parental alleles involved in 
the cross (Kitani and Olive, 1967, 1969), when a conversion occurs between only a pair of homologous chromatids of a bivalent. Gene conversion of S. fimicola is this 2-chromatid event with very few exceptions (Kitani, 1962, 1978), unlike Ascoborus immersus where 4-chromatid event is usual (Lamb and Wickramaratne, 1973; Paquette, 1978; Kitani, 1978).

Among the ascus types, the $M$ type is phenotypically identical to a tetratype from reciprocal recombination. Therefore, the population of $\mathbf{M}$ type asci expands proportionally by the presence of reciprocal recombination. And this increased portion is represented by $\mathbf{E}^{a \times b}$ variant in the formula, $\mathrm{I}^{a \times b}=0^{a}+0^{b}+E^{a \times b}$, explained in the following section.

III Fine analysis of interallelic cross conversion frequencies of the $i$-locus using corrected one-point cross conversion frequencies

\section{1) Procedure of analysis}

All the data on aberrant segregation asci subjected to the present finer reexamination are from Kitani (1982). The method to correct the one-point cross conversion frequencies is described in Table 3 of Kitani and Olive (1967). The actual equations used here are: $b^{2}=(a b \times b c) \div\left(\sqrt{a^{2}} \times \sqrt{c^{2}}\right)$, for aberrant 4:4 type asci; and ac $=\sqrt{\mathrm{a}^{2}} \times \sqrt{\mathrm{c}^{2}}$, for the normal 4:4 type asci appeared through a particular combination of base correction modes. Symbols for the modes of base correction can be referred from Table 3 and Table 4 of Kitani and Olive (1967).

The basic formula, $\mathrm{I}^{\mathrm{a} \times \mathrm{b}}=\mathrm{O}^{\mathrm{a}}+\mathrm{O}^{\mathrm{b}}+\mathrm{E}^{\mathrm{a} \times \mathrm{b}}$, is used here to analyse the nature of the $i$-locus interallelic cross corversion frequencies as well as to estimate the presence and its extent of intralocus reciprocal recombination. In this formula, I-variant means the interallelic cross conversion frequency between the alleles a and $\mathrm{b}$; $\mathrm{O}$-variants mean the one-point cross conversion frequencies of the alleles a and $b$ employed in the cross; and $\mathrm{E}$-variant means an extra frequency onver the added value of $\mathrm{O}^{\mathrm{a}}$ and $\mathrm{O}^{\mathrm{b}}$.

For the convenience of comparative examination, all the results from various aspects are shown in Tables 1 and 2 of the same title. Variant $\mathrm{O}^{i_{1}}$ to $\mathrm{O}^{i_{10}}$ in the upper heading are the one-point cross conversion frequencies of the indicated alleles of indigo spore colour expression; corrected values and uncorrected values (in parentheses; refer to Kitani, 1982) are shown together. Corrected frequencies with an asterisk are significantly larger than the other seemingly uniform one-point cross conversion frequencies, and these are discussed in the text. Since the $i$-locus alleles aer phenotypically indistinguishable in interallelic crosses between the alleles of same colour expression, crosses were made between indigo and blue.

To examine the addtive nature of the $i$-locus interallelic cross conversion frequencies to those of the parental alleles one-point cross conversion frequencies, five different values are compared at the category a) in each box of the tables. 
Table 1. Relationaship between one-point cross and interallelic cross conversion frequencies in the $i$-locus of Sordaria fimicola.

I. With single values of one-point cross convesion frequency ${ }^{\text {a) }}$

\begin{tabular}{|c|c|c|c|c|c|c|}
\hline \multicolumn{2}{|c|}{$\begin{array}{l}\text { Expression } \\
\text { Ex- indigo } \\
\text { pression } \\
\text { blue }\end{array}$} & $\begin{array}{c}i_{1} \\
\mathrm{O}^{i_{1}} ; \underset{(8.03)}{10.4}\end{array}$ & $\begin{array}{c}i_{2} \\
\mathrm{O}^{i_{2} ;} \underset{(7.95)}{* 20.8}\end{array}$ & $\begin{array}{c}i_{5} \\
0^{i_{5} ; 12.0} \frac{18.96)}{(8.9)}\end{array}$ & $\begin{array}{c}i_{8} \\
\mathrm{o}^{i_{8} ; 13.4} \\
(10.59)\end{array}$ & $\begin{array}{c}i_{10} \\
\mathrm{O}^{i_{10}} ; \underset{(9.80)}{13.4}\end{array}$ \\
\hline \multirow{5}{*}{$\begin{array}{c}b l u_{3} \\
\mathrm{o}^{b l u_{3}} \\
\quad \begin{array}{l}13 . \dot{4} \\
(9.81)\end{array}\end{array}$} & a) & 12.8 & 20.1 & 12.8 & 12.8 & 12.8 \\
\hline & b) & 31.0 & $32.1 *$ & 25.7 & $22.5 *$ & 28.8 \\
\hline & c) & 18.2 & 12.0 & 12.9 & 9.7 & 16.0 \\
\hline & d) & 3.1 & 6.1 & 4.0 & 8.5 & 2.2 \\
\hline & e) & 7.9 & 0.8 & 1.4 & 1.6 & 2.6 \\
\hline \multirow{5}{*}{$\begin{array}{l}b l u_{4} \\
0^{b l u_{4}} \\
* 19.3 \\
(13.90)\end{array}$} & a) & 20.1 & 20.1 & 20.1 & 20.1 & 20.1 \\
\hline & b) & 28.4 & $22.2 *$ & $24.9 *$ & 34.4 & 35.5 \\
\hline & c) & 8.3 & 2.1 & 4.8 & 14.3 & 15.4 \\
\hline & d) & 7.5 & 7.5 & 7.6 & 3.1 & 4.4 \\
\hline & e) & 5.3 & 1.8 & 2.9 & 2.5 & 5.9 \\
\hline \multirow{5}{*}{$\begin{array}{l}b l u_{6} \\
\text { rec }^{-} \\
\mathrm{o}^{b l u_{6}} \\
\quad 12.9 \\
(9.27)\end{array}$} & a) & 12.8 & 20.1 & 12.8 & 12.8 & 12.8 \\
\hline & b) & 36.1 & $22.2^{*}$ & $22.3^{*}$ & 29.3 & 30.3 \\
\hline & c)] & 23.3 & 2.1 & 9.5 & 16.5 & 17.5 \\
\hline & d) & -4.5 & 6.0 & 3.0 & -0.2 & -1.5 \\
\hline & e) & 8.2 & 1.0 & 1.4 & 0.4 & 6.4 \\
\hline \multirow{5}{*}{$\begin{array}{l}b l u_{7} \\
0^{b l u_{7}} \\
12.7 \\
(9.22)\end{array}$} & a) & 12.8 & 20.1 & 12.8 & 12.8 & 12.8 \\
\hline & b) & 30.6 & $36.0 *$ & 30.9 & $24.1 *$ & 31.5 \\
\hline & c) & 17.8 & 15.9 & 17.3 & 11.3 & 18.7 \\
\hline & d) & -5.9 & -5.3 & -3.2 & 0.5 & -4.9 \\
\hline & e) & 5.5 & 0.4 & 1.4 & 1.3 & 5.4 \\
\hline \multirow{5}{*}{$\begin{array}{l}b l u_{9} \\
o^{b l u_{9}} \\
\quad 13.4 \\
\quad(9.99)\end{array}$} & a) & 12.8 & 20.1 & 12.8 & 12.8 & 12.8 \\
\hline & b) & 37.8 & $22.8^{*}$ & 24.5 & 29.9 & $21.8^{*}$ \\
\hline & c) & 25.0 & 2.7 & 11.7 & 17.1 & 8.9 \\
\hline & d) & -2.8 & 9.7 & 6.2 & -9 & 7.5 \\
\hline & e) & 5.8 & 0.4 & 0.5 & 1.8 & 3.5 \\
\hline
\end{tabular}

a) Frequencies are at $10^{-3}$.

Here, the values 12.8 and 20.1 represent $0^{\mathrm{m}} 1$ and $\mathrm{O}^{\mathrm{m}} 2$, respectively, which are the mean values of one-point cross conversion frequencies (corrected) of all the $i$-locus alleles excluding $i_{2}$ and $b l u_{4}$ and of the alleles $i_{2}$ and $b l u_{4}$, respectively. Values 25.6, 32.9 and 40.2 represent $2 \times 0^{\mathrm{m}} 1,0^{\mathrm{m}} 1+0^{\mathrm{m}} 2$ and $2 \times 0^{\mathrm{m}} 2$, respectively.

Fit or not as the interallelic cross conversion frequencies of these single or added one-point cross conversion frequencies is examined in Tables 1 and 2 , in which each box represents each interallelic cross indicated by the headings. 
Table 2. Relationaship between one-point cross and interallelic cross oonversion frequencies in the $i$-locus of Sordaria fimicola.

II. With additive values of one-point cross conversion freqiency ${ }^{\text {a) }}$

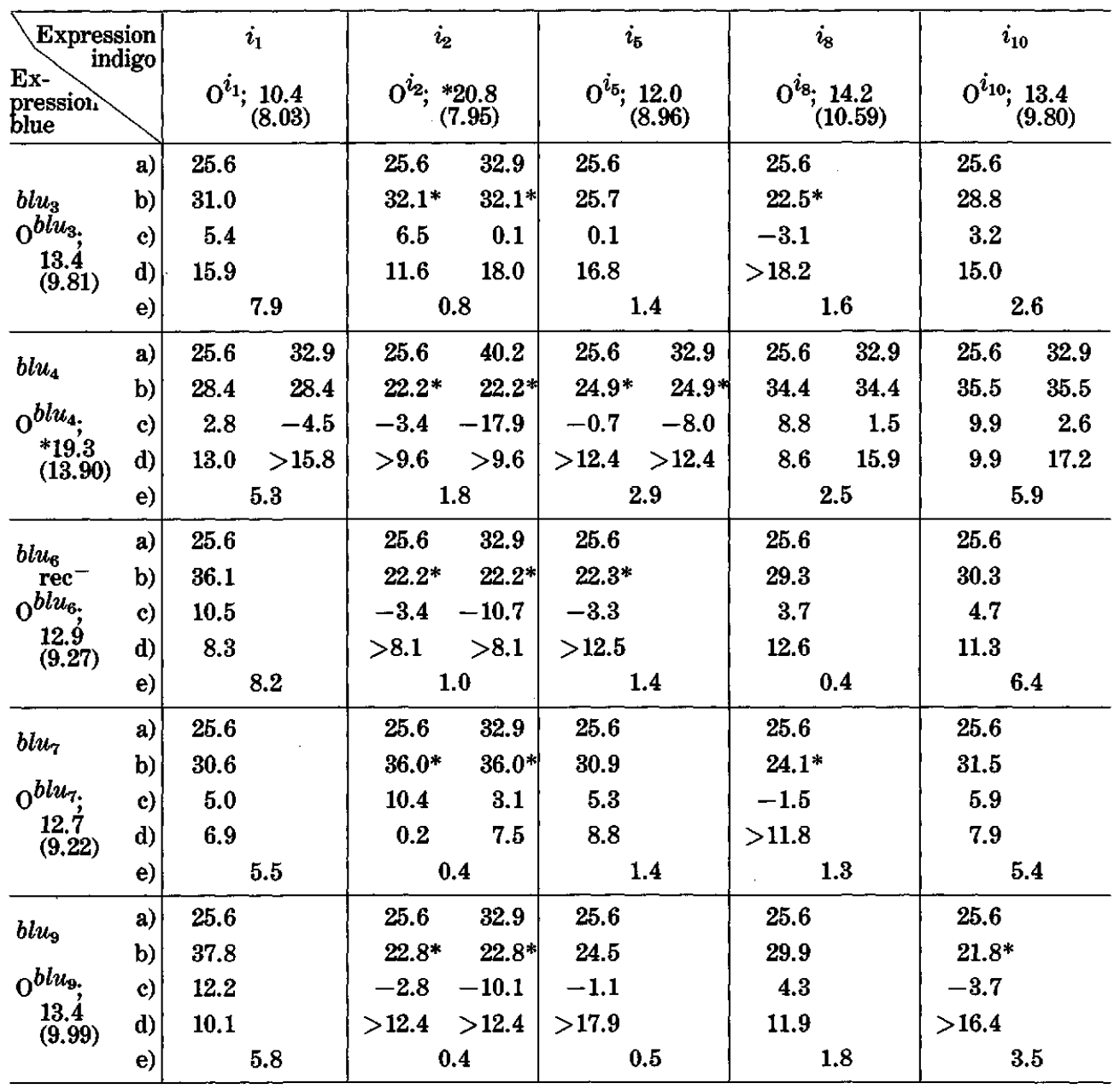

a) Frequencies are at $10^{-3}$.

Values given in category $\mathrm{b}$ ) in the tables are the actually observed $\mathrm{I}^{\mathrm{a} \times \mathrm{b}}$ variants for each of the respective interallelic cross, and all of them are shorter than the real because of the inevitable overlooking of conversion ascus types $E$ and $F$ which show indigo:blue spore colour ratio at 4:4 (see Tables 4 and 5 of Kitani, 1982, as well as Figs. 3 and 4 and Table 1 of Kitani and Olive, 1969). Band intensities for these ascus types vary in the respective conversion spectra, depending upon the modes of one-point cross conversion spectra of the parental alleles (refer to Kitani and Olive, 1969). Therefore, the degree of the above stated shortage varies from 
negligible to significant. Values of category b) marked with asterisks in Tables 1 and 2 are considered through the parental conversion spectra to be significantly shorter than the real (corrected values are not shown in the tables, because the estimated values for aberrant and normal 4:4 conversion asci are used for these calculations). Effects of this shortage in category b) on evaluation of the values shown in categories c) and d) are explained at the appropriate places.

Values shown in category c) are the results of subtraction of a a)-value from a b)-value in each of the crosses, and represent the variants $E^{a \times b}$. In the boxes of Tables 1 and 2 where the b)-values are considered to be substantially shorter than the real (marked with an asterisk), the actual values for category c) must be substantially smaller than those appeared. When the value in category c) is substantial at the positive side, it suggests the substantial presence of intra- $i$ locus reciprocal recombination. And, when the c)-value is substantial at the negative side in the boxes where the b)-value is not marked with an asterisk, it means that the $\mathrm{O}$-valiant given in category a) is untenably large; while in the boxes with asterisked $b$ )-value, $c$ )-value is allowed to be negative up to the amount of overlooked asci of ascus types $\mathrm{E}$ and $\mathrm{F}$.

Values shown in category d) represent the sizes of the remaining conversion ascus populations showing ascospore colour ratio of $2+, 2 \mathrm{i}, 4 \mathrm{~b}$ (ascus types $\mathrm{M}$ and 0 ) after removing the respective E-values (a part of $M$ ascus population) as the genuine reciprocal recombinants (refer to Figs. 3 and 4 and Table 2 of Kitani and Olive, 1969, and Table 4 of Kitani, 1982). In the boxes where b)-values are marked with asterisks, d)-values are including excess portions corresponding to the sizes of overlooked ascus types $E$ and $F$. When the value in category $d$ ) is too small as to represent the expected ascus population for ascus types $M$ and $O$ or at the negative side, it means that the $O$-valiant tested in category a) is untenably small.

By restricting the allowance of the 0-variant used in category a) in each box of the Tables from both flanks, as explained above, by the requirments in categories c) and d), the most probable nature or value can be determined for each conversion frequency of the $i$-locus interallelic crosses.

Values shown in category e) are the estimated population sizes for ascus types $\mathrm{M}$ and $\mathrm{O}$ in each of the $i$-locus interallelic crosses. For the estimation of $\mathrm{M}$ type population, the population of normal 4:4 conversion asci in one-point cross conversion spectrum of each blue allele is multiplied with the sum of the one-half values for normal $4: 4$ and $5+3 \mathrm{~m}$ asci and the full population of $6+: 2 \mathrm{~m}$ asci in one-point cross conversion spectrum of each indigo allele. For the estimation of 0 type population, the proportion of aberrant 4:4 asci of one-point cross conversion spectrum of each blue allele is multiplied with the proportion of $6+2 \mathrm{~m}$ asci of the indigo allele spectrum. For the foundation of these estimation, refer to Fig. 4 of Kitani and Olive (1969). Although these values are referred to judge the fit or not of d)-values, consequently of the respective a)-values, these are not solid 
values because the used proportions for normal and aberrant 4:4 ascus types are estimated values.

\section{2) Results of the reexamination}

It was already reported that conversion frequencies of the $i$-locus interallelic crosses were not the same as the apparently uniform one-point cross conversion frequencies (refer to Kitani, 1982, and Kitani and Olive, 1967, 1969, for the difference of the loci $g$ and $i$ in conversion frequencies), but these varied, ranging from near double of one-point cross conversion frequencies to still higher values (kitani, 1982). The reason for the $i$-locus interallelic cross conversion frequencies being the sums of the respective one-point cross conversion frequencies was explained as that the $g$-locus is composed of a single conversion unit, "convertron", while the $i$-locus is composed of multiple conversion units (Kitani, 1982). The nature of extra conversion frequencies sometimes found in the $i$-locus interallelic crosses, as the portions exceeding double value of one-point cross conversion frequencies, was explained as to indicate the presence and its extent of intra- $i$ locus genuine reciprocal recombination (Kitani, 1982).

The present finer analysis using the corrected one-point cross conversion frequencies generally confirmed and strengthened the above two concepts, as well as raising a possibility of that the size of conversion units may not be uniform.

\section{2-A) Corrected one-point cross conversion frequencies}

As shown in the top headings of Tables 1 and 2, corrected conversion frequencies for the alleles of indigo ascospore colour expression, $i_{1}, i_{2}, i_{5}, i_{10}$, appeared as follows; 10.4, 20.8, 12.0,14.2 and 13.4, all at $10^{-3}$, respectively. As shown in the left-hand side headings of the Tables, corrected conversion frequencies for the alleles of blue ascospore colour expression, $b l u_{3}, b l u_{4}, b l u_{6} \mathrm{rec}^{-}, b l u_{7}$ and $b l u_{9}$ appeared as follows; $13.4,19.3,12.9,12.7$ and 13.4 , respectively.

Different from the concept of Kitani (1982), developed through the uncorrected frequencies, given in parentheses in the headings, the corrected frequencies appeared in two different sizes: around $20 \times 10^{-3}$ and around $13 \times 10^{-3}$. Although this difference is substantial in consideration of the sample size, statistical tests are not given here, because the corrected values are estimated ones. However, it seems reasonable to consider that the conversion units in which each of tese 10 alleles locates are not uniform in size, but consist of at least two groups of large and small units. Based on this fact, two mean values, $0^{\mathrm{m}} 1=12.8 \times 10^{-3}$ and $0^{\mathrm{m}} 2=20.1 \times 10^{-3}$ are used to represent all the $i$-locus alleles excuding $i_{2}$ and $b l u_{4}$, and exclusively $i_{2}$ and $b l u_{4}$, respectively.

\section{2-B) Composition of interallelic cross conversion frequencies}

In the $i$-locus conversion analysis of Kitani (1982), the base of conversion frequencies in interallelic corsses was tentatively considered as the sum of 
one-point cross conversion frequencies of the respective alleles employed in the cross. Since the raw conversion frequencies looked uniform, it was concluded in Kitani (1982), that the minimum (basic) conversion frequency for the $i$-locus interallelic crosses was double value of the uniform conversion frequency of one-point crosses.

In the present analysis using the corrected frequencies, fit or not of the sampled frequencies with the actual data was determined by a set of restricting factors apocalyptic in c)- and d)-values in Tables 1 and 2. As explained in Procedure of analysis, the frequency sampled in category a) is untenably large when the c)-value is substantial at the negative side, while the frequency sampled is untenably small when the d)-value is too small or at the negative side. These restrictions from both flanks in Tables 1 and 2 ultimately revealed that the frequency $25.6 \times 10^{-3}=2 \times 0^{\mathrm{m}} 1 \mathrm{might}$ represent the basic (or minimal) conversion frequency in interallelic crosses of the $i$-locus.

(i) Tests on single values of one-point cross conversion frequencies

In Table 1, two frequency values $12.8\left(0^{\mathrm{m}} 1\right)$ and $20.1\left(0^{\mathrm{m}} 2\right)$ are examined. Leaving off the boxes with $b$ )-values amrked with asterisks, the d)-values in the following crosses appeared negative; $i_{1} \times b l u_{6}, i_{1} \times b l u_{7}, i_{1} \times b l u_{9}, i_{5} \times b l u_{7}$, $i_{8} \times b l u_{6}, i_{8} \times b l u_{9}, i_{10} \times b l u_{6}$ and $i_{10} \times b l u_{7}$. This indicates that the tested frequency, 12.8, is untenably small as the conversion frequency of three interallelic crosses.

Through the size comparison between the values in categeries d) and e), the a)-values, both 12.8 and 20.1, are judged to be too small as the conversion frequency of the following interallelic crosses; $i_{1} \times b l u_{3}, i_{1} \times b l u_{4}, i_{8} \times b l u_{4}$, $i_{10} \times b l u_{3}$ and $i_{10} \times b l u_{4}$.

In the boxes of which b)-values are with asterisks and are significantly shorter than the real, the d)-values appeared are consequently longer than the real (around or over 10; see Procedure of analysis). Even under such conditions, an interallelic cross, $i_{2} \times b l u_{7}$, shows a negative d)-value; hence, the a)-value 20.1 is untenably small. And, in giving considerations on the real sizes of d)-values, the a)-values are indirectly judged to be too small in the crosses $i_{2} \times b l u_{3}, i_{2} \times b l u_{4}$, $i_{2} \times b l u_{6}, i_{5} \times b l u_{4}, i_{8} \times b l u_{7}$ and $i_{10} \times b l u_{9}$. Thus only three interallelic crosses, $i_{2} \times b l u_{9}, i_{5} \times b l u_{3}$ and $i_{5} \times b l u_{9}$, out of the 25 crosses, remained somewhat ambiguous.

From the above information with Table 1, it is thus confirmed that the basic conversion frequency (without including genuine reciprocal recombination) of the $i$-locus interallelic crosses is not the same as the one-point cross convesion frequency, but is far larger.

(ii) Determination of appropriate additive value of one-point cross conversion frequencies for interallelic cross basic conversion frequency 
In Table 2, three additive values, $25.6\left(2 \times 0^{\mathrm{m}} 1\right), 32.9\left(0^{\mathrm{m}} 1+\mathrm{O}^{\mathrm{m}} 2\right)$ and 40.2 $\left(2 \times \mathrm{O}^{\mathrm{m}} 2\right)$ are tested through the examination of the respective $\left.\mathrm{c}\right)$-values. When there is no genuine intra-i-locus recombination, the respective c)-value can be nil but cannot be negative in the boxes where the b)-values are not marked with asterisks; if significant at the negative side, it means that the a)-value tested is untenably large. While in the boxes where the b)-values are marked with asterisks c)-values are allowed to be negative in small extent.

At first, regarding the a)-value of 25.6, all but one of interallelic crosses between the alleles of smaller one-point cross conversion frequencies [excluding the boxes with asterisk-marked b)-values] showed good fit. These crosses are $i_{1} \times b l u_{3}, \quad i_{1} \times b l u_{6}, \quad i_{1} \times b l u_{7}, \quad i_{1} \times b l u_{9}, \quad i_{5} \times b l u_{3}, \quad i_{5} \times b l u_{7}, \quad i_{8} \times b l u_{9}, \quad i_{10} \times b l u_{3}$, $i_{10} \times b l u_{6}$ and $i_{10} \times b l u_{7}$. Since all the $\mathrm{b}$ )-values are more or less shorter than the real, the small negative c)-value, -1.1 , in the cross $i_{5} \times b l u_{9}$ would be within the range of allowance.

In the boxes with astersk-marked b)-values, all the interallelic crosses, $i_{5} \times b l u_{6}$, $i_{8} \times b l u_{3}, i_{8} \times b l u_{7}$ and $i_{10} \times b l u_{9}$ showed small negative values. These negative values are well within the range of allowance in consideration of the conversion spectra of the parental alleles. All the above results suggest that the basic conversion frequency for the crosses between any of the $i$-locus alleles, excluding $i_{2}$ and $b l u_{4}$, is 25.6 , which is $2 \times 0^{\mathrm{m}} 1$.

Secondly, regarding the alternative a)-values, $32.9=0^{\mathrm{m}_{1}} 1+\mathrm{O}^{\mathrm{m}} 2$ and $40.2=2 \times 0^{\mathrm{m}} 2$, these are compared with $25.6=2 \times 0^{\mathrm{m}_{1}}$ in the interallelic crosses between $i_{2}$ or $b l u_{4}$ and other alleles. At the time of such comparisons, judgement through the sizes of c)-values is helped by the size relationship between the respective $d$ )- and e)- values.

In the boxes with unmarked b)-values the c)-values in a cross $0^{i_{1}} \times b_{l} u_{4}$ are positive when the a)-value is 25.6 , and negative when the a)-value is 32.9 . This means that $2 \times 0^{m_{1}}$ is more suitable than $0^{m_{1}}+\mathrm{O}^{\mathrm{m}} 2$ regardless of that the parental alleles are the members of mean $1\left({ }^{\mathrm{m}} 1\right)$ and mean $2\left({ }^{\mathrm{m}} 2\right)$, respectively. In other crosses of the group, $i_{8} \times b l u_{4}$ and $i_{10} \times b l u_{4}$, both of the b)-values in a box are positive. But, the differences between the $d$ )- and e)-values are significantly closer when the a)-value is 25.6. From this fact too, the value $25.6=2 \times 0^{\mathrm{m}} 1$ is recommended at the basic conversion frequency of these crosses.

In the boxes with the marked b)-values, c)-values are allowed to be negative, if small. In the crosses $i_{2} \times b l u_{3}$ and $i_{2} \times b l u_{7}, b$ )-values are both positive for both of the a)-values. However, 25.6 is more favoured than the other bacause of the smaller differences between the respective $d$ )- and e)-values. In the crosses $i_{2} \times b l u_{6}, i_{2} \times b l u_{9}$ and $\left.i_{5} \times b l u_{4}, c\right)$-values are all at the negative side. Since to be too large at the negative side is not allowed, the a)-value of $25.6=2 \times 0^{\mathrm{m}} 1$ is also favoured in all these three crosses. In a single cross between the alleles of larger one-point cross conversion frequencies, $i_{2} \times b l u_{4}$, the negative amount of the c)-value is too large for the respective a)-value of $40.2=2 \times \mathrm{O}^{\mathrm{m}} 2$, even though the 
other b)-value too is at the negative side.

Combining all the above observations, the basic conversion frequency for the $i$-locus interallelic crosses is likely to be a single value, $25.6=2 \times 0^{\mathrm{m}} 1$, even if one or both of the parents belonged to the allele group of larger one-point cross conversion frequency. Applying this to the formula $\mathrm{I}^{\mathbf{a} \times \mathbf{b}}=\mathrm{O}^{\mathbf{a}} \times \mathrm{O}^{\mathbf{b}}+\mathrm{E}^{\mathrm{a} \times \mathrm{b}}$, given and explained in Procedure of analysis, it can be now practically modified as: $\mathrm{I}^{\mathrm{a} \times \mathrm{b}}=2 \times 0^{\mathrm{m}} 1+\mathrm{E}^{\mathrm{a} \times \mathrm{b}}$.

\section{3) Intralocus geniune reciprocal recombination}

If the basic conversion frequency for the $i$-locus interallelic crosses is a uniform $2 \times 0^{\mathrm{m}} 1\left(=25.6 \times 10^{-3}\right)$ or sometimes a little larger $0^{\mathrm{m}} 1+\mathrm{O}^{\mathrm{m}} 2\left(=32.9 \times 10^{-3}\right)$, the surplus variant, $\mathrm{E}^{\mathrm{a} \times \mathrm{b}}$, in the formula $\mathrm{I}^{\mathrm{a} \times \mathrm{b}}=\mathrm{O}^{\mathrm{a}}+\mathrm{O}^{\mathrm{b}}+\mathrm{E}^{\mathrm{a} \times \mathrm{b}}$ represents the amount of tetratype asci recorded as a conversion ascus type $M$. And this is the amount of intra- $i$-locus genuine reciprocal recombination in each cross. In Table 2 , this variant is represented by the c)-values at the conditions of a little bit shorter than the real [when the b)-value of the box is not asterisked] to somewhat shorter than the real [when the b)-value of the box is asterisked].

The actual c)-values, regarding the fixed a)-value of 25.6, vary from insignificantly negative to slightly over $12 \times 10^{-3}$. This suggests two facts: (1) genuine reciprocal recombination occurs in the $i$-locus of Sordaria fimicola, and (2) the frequency of such recombination varies from cross to cross, ranging from nil to over $10 \times 10^{-3}$.

Since conversion frequencies of the $i$-locus are roughly uniform in one-point crosses, the basic conversion frequency for interallelic crosses is now known to be double of the one point cross conversion frequencies, and the frequency of reciprocal recombination event varies from cross to cross, it is now judged that intra- $i$-locus reciprocal recombination takes place at the intervals (or joints) of the conversion units, convetrons. If an interallelic cross involves only the adjacent convertrons with little or no interval, there will be little or no reciprocal recombination. While, if there are number of convertron intervals between the respective convertrons of the cross, there will be multiple chances for the recombination event.

\section{Discussion}

\section{1) On the results of re-examination}

The prosent detailed analysis on the $i$-locus interallelic cross conversion frequencies, based on the corrected one-point cross conversion frequencies, confirmed or modified the previous findings in Kitani (1982), in the following points. 1. Corrected one-point cross conversion frequencies of the $i$-locus alleles are likely to fall into two groups, about $12.8 \times 10^{-3}$ and $20.1 \times 10^{-3}$.

2. The basic conversion frequency of the $i$-locus interallelic crosses is around 
$26 \times 10^{-3}$ (although a possibility to be about $33 \times 10^{-3}$ cannot be excluded for some particular crosses.) This is twice the value of the smaller of the above mentioned two mean values for one-point cross conversion frequencies.

3. The presence and the extent of intra-i-locus genuine reciprocal recombination are recongnized in interallelic crosses as the presence and the extent, respectively, of surplus conversion frequencies over the above mentioned basic conversion frequency of $26 \times 10^{-3}$.

\section{2) General agreements in Sordaria gene conversion}

Combining these present findings with the characteristic features of the $g$-locus gene conversion reviewed in Section II, the general agreements in gene conversion and reciprocal crossing over in Sordaria fimicola can be drawn as follows.

1. Each gene locus has either a single unit or multiple units of "convertron", the conversion unit.

2. All the alleles in a single convertron share the same one-point cross conversion frequency.

3. Conversion frequency in an interallelic cross between any alleles in a single convertron is the same as the one-point cross conversion frequency of the respective convertron.

4. Basic conversion frequency of an interallelic cross between any alleles of different convertrons is or near the sum of one-point cross conversion frequencies of the respective convertrons.

5. Every basic conversion frequency of the interallelic crosses in items 3 and 4 involves a population of apparent reciprocal recombinats originated through gene conversion, but does not involve genuine reciprocal recombinants.

6. Surplus conversion frequency, if found in an interallelic cross of item 4, indicates the presence, and represents the extent, of genuine intralocus reciprocal recombination.

7. Flanking marker exchanges occur in association with gene conversion events through an integrated mechanism both in one-point and interallelic crosses at the same frequency of $50 \%$, if double exchanges are not accounted for.

\section{3) Concepts regarding reciprocal recombinations}

From the above agreements established directly from the observed phenomena, the following concepts are resulted regarding reciprocal recombinations:

a) Conversion-associated flanking marker exchanges are reciprocal recombinations regarding the flankling markers if the conversion event is ignored or indistringuishable (like the case of negative interference in Aspergillus.) But the frequency of such recombinations is constant at a half of the respective conversion frequency; irrespective to the map distance between the flanking markers.

b) Intralocus reciprocal recombination occurs somewhere between the adjacent convertrons and the frequency is likely to be proportional to the map distance, 
when the respective alleles are interposed with multiple convertrons.

c) Although there is yet no information to connect intralocus reciprocal recombination to classical interlocus crossing over directly, no evidence to discriminate these, either. But, if a particular base sequence is determined to be present in the $i$-locus but absent in the $g$-locus, this sequence would be directly related to the position of recombination (or a recombination hot-spot.) By extending the search for such a sequence beyond the locus boundary, the nature of, and the spots for, the classical crossing over would be better understood.

4) The relation of the concepts developed in Sordaria research with MeselsonWhitehouse-Fogel models

The above stated concepts on reciprocal recombination with regards to gene conversion are quite different from the ones now widely accepted, such as the models of Meselson and Radding (1975), and Fogel et al. (1979). The latter concepts are based on the stand point of base correction within the DNA heteroduplex of Holliday-Whitehouse model (Holliday, 1964; Whitehouse, 1964; Whitehouse, 1963) which relied on the data of S. fimicola.

In the background of causing the above conceptual difference, there exist critical differences in the nature of the working systems and the range of implication can cover with scientific confidence. At the side of S. fimicola, representing the materials for octad analysis, the working system has been the closest so far established to fulfil the critical requirments in the theoretically ideal octad analysis. And this system is carefully organized to analyse each octad comprehensively regarding various events through meiosis. Therefore, Sordariasystem has been most gifited in, and contributed to, establishing a wide-ranged bird-eye view comprehensively on the "results" of meiosis in detail. To do this, it has been necessary to determine the genotype of each octad through orderly dissection of asci and culturing. Naturally, the analysable frequency level is restricted for such an elaborated way, as an compensation to the finely accurate information. Thus, the frequency order allowed for the analysis of events in this system has heen $10^{-3}$ to $10^{-4}$. Very fortunately, however, the events as the direct results of meiosis (exclusively eukaryotic phenomenon), such as gene conversion, flanking marker exchange and even for the map distance of the closest flanking amrker, etc. are in the range mentioned above.

In some materials short to fulfil the rigid requirments for the ideal octad analysis, such as having eight ascospores but not in order, lacking suitable flanking amrkers, lacking vasibly distingishable ascospore phenotypes or having only four ascospores, etc., studies have been advenced to analyse random spores or offspring subjecting the phenomena of molecular level. This method is gifted in, and contributed to, establishing the deeply penetrating insight into the individual "process" of events showing far lower frequencies than the other.

By examining the above views together, one on the direct results of meiosis and 
the other on the fine events during meiotic process, commanding one view by the other connot be done, and should not be attempted. Because, the phenomena that these views examine are at the separate phases and at different frequency levels. From the side of octad analysis, occurrence of events at the frequencies lower than $10^{-4}$ can make little influence on the results at the order of $10^{-3}$. In contrast, from the side of random product molecular analysis, the view from Sordaria octad analysis is Shangri-La without a molecular proof.

5) Bridging the gap between the concepts from the Sordaria data and the models based on excision repair synthesis

Researchers on the mechanism of eukaryotic recombination should eventually bridge the above two viewpoints. The only way to do this would be an evenfooted cooperation by the researchers of both schools. Now a prospect to determine a particular base sequence for intra- (and possibly also inter-) locus recombination, from the comparison of total base sequences of the loci $g$ and $i$, is raised by the present report. If this can be done by the above mentioned cooperation, most of the tedious molecular work in bridging the gap could be largely bypassed.

\section{6) Summarized process for gene conversion and related phenomena}

Due mainly to the entire lack of a similar example of the "single convertron gene locus" like the $g$-locus of $S$. fimicola, in the materials so far used in genetic recombination research, the mechanism of gene conversion and related phenomena discussed individually in Kitani and Olive $(1967,1969)$, and Kitani (1978a, 1978b), have not acquired general understanding. And the once very famous implication of Holliday (1964) and Whitehouse (1963) in now largely overlooked. However, since the presence and the role of "convertron" became clear through the present re-examination (section III), it may be appropriate to summerize a comprehensive scheme for the sequential process of gene conversion and the related phenomena.

The sequence is: (1) Simulataneous and mutual formation of a pair of hybrid DNA (heteroduplex) between a pair (not two pairs) of the homologous chromatide of a bivalent chromosome; (2) Presence or absence of the flanking marker exchange is differentiated at the begining of heteroduplex formation as illustrated in Kitani and Olive (1969) and Kitani (1978); (3) Mismatch base correction takes place independently in each of the hybrid DNA molecules, but frequency and direction of the base correction are influenced by the allele's preference; and (4) When one or both of the hybrid DNA molecules remained uncorrected until the first postmeiotic S-phase, a 5:3 or an aberrant 4:4 segregation ascus appeared.

7) Times for initiation of gene conversion and reciprocal recombinations

Genetic recombination in eukaryotes cannot be established without synapsis 
between the homologues, and the general underestanding for the time of homologous pairing is still limited in meiotic prophase. This is the reason why Stern's far advanced pioneering discovery of twin-spot somatic recombination (stern, $1936,1969)$ is not appropriately credited, and the discussions for other somatic recombinations (Pontecoryo et al., 1953; Pritchard, 1960; Putramena, 1967a, 1967b; Pontecorvo and Käfer, 1958), somatic gene convarison (Roman and Fabre. 1983) are all done under the hesitative tone. However, since the publication on a possibility of general and regular occurrence of somatic homologous pairings in every interphase (Kitani, 1963), numerous findings to support this concept appeared both in premeiotic (ultimate) interphase (Chauhan and Abel, 1968; Bowman and Rajhathy, 1977; Maguir, 1983) and somatic interphase (Feldman et al., 1966; Rao and Natarajan, 1967; Comings, 1968; Wagenaar, 1969; Rieger, et al., 1973; Stack and Clark, 1973; Yoshida and Yamaguchi, 1973; Colombera and Cary, 1973; Vig, 1973; Diaz and Lewis, 1975; Svidchenko, 1975; Crozier, 1975; Singh, Röbbelen and Okamoto, 1976; van Heemert, 1977; and Lacadena and Ferrer, 1978).

Gene conversion and associated flanking marker exchanges initiate as an integrated mechanism after S-phase (Presence of post-meiotic segregation and 4chromatid type conversion restricts the timing as this) of both somatic and premeiotic interphase. Other kinds of recombination event can initiate any time throughout interphase by themselves, or in addition to gene conversion.

\section{Conclusion}

The $g$-locus of $S$. fimicola shows a uniform conversion frequency in all the tested conditions, while the conversion frequencies are different in the $i$-locus between one-point crosses and interallelic crosses. Various phenomena that appear with the $g$-locus conversion suggest the absence of genuine reciprocal refombination in this locus. The present reexamination using corrected one-point cross conversion frequencies confirmed these. Gene conversion is a separate event from genuine intralocus reciprocal recombination, and involves DNA heteroduplex formation throughout a convertron and mismatch base correction instead of excision-repair synthesis of DNA. Genuine intralocus reciprocal recombination occurs between the adjacent convertrons in a locus of multiple convertron composition.

Sordaria stock culture for the gene conversion research have been deposited with both FGSC and ATCC. The research on the $i$-locus and maintenance of Sordaria stocks would not have been done without the supports of Grant-in-Aid No. 448007 from the Ministry of Education, and Grant 78-1904 from the Toray Science Foundation.

\section{REFERENCES}

BollaG, R. J. and LiSKAY, R. M. (1988) Conservative intrachromosomal recombination between in- 
verted repeats in mouse cells: Association between reciprocal exchange and gene conversion. Genetics 119, 161-169.

Bowman, J. G. and RAJHATHY, T. (1977) Function of chromocenters in premeiotic interphase of Secale cereale and its possible relationship to chromosome pairing. Canad. J. Genet. Cytol. 19, 313-321.

Chauhan, K. P. S. and ABeL, W. O. (1968) Evidence for the association of homologous chromosomes during premeiotic stages in Impatiens and Salvia. Chromosoma 25, 297-302.

Clombera, D. (1973) Somatic pairing in Botryllus schlosseri Pallas (Ascidiacea). Caryologia 26, 27-33.

Comings, D. E. (1968) The rationale for an ordered arrangement of chromatin in the interphase nucleus. Am. J. Hum. Genet. 20, 440-460.

Crozier, R. H. (1975) Centromeric region pairing in somatic chromosomes of the midge Chironomus tentans (Diptera: Chironomidae). Canad. J. Genet. Cytol. 17, 193-195.

DiAZ, G. and LEwIS, K. R. (1975) Interphase chromosome arrangement in Anopheles atroparvus. Chromosoma 52, 27-35.

Feldman, M., Mell-Sampayo, T, and Seare, E. R. (1966) Somatic association in Triticum aestivum. Genetics 56, 1192-1199.

Fogel, S., Mortimer, R., Lusnak, K., and Tavares, F. (1979) Meiotic gene conversion: a signal of the basic recombination event in yeast. Cold Spr. Harbor Symp. Quant. Biol. 43, 1325-1341.

Holliday, R. (1964) A mechanism for gene conversion in fungi. Genet. Res. (Camb.) 5, $282-304$.

KITANI, Y. (1962) Three kinds of transreplication in Sordaria fimicola. Jpn. J. Genet. 37, 131-146.

KITANI, Y. (1963) Orientation, arrangement and association of somatic chromsomes. Jpn. J. Gent. 38, 244-256.

KITANI, Y. (1978) Absence of interference in association with gene conversion in Sordaria fimicola, and presence of interference in association with ordinary recombination. Genetics 89, 467-497.

KITANI, Y. (1978) Aberrant 4:4 segregation. Jpn. J. Genet. 53, 301-308.

KITANI, Y. (1982) Characteristics of gene conversion and Rec factor of the $i$ locus of Sordaria fimicola. Jpn. J. Genet. 57, 467-481.

KITANI, Y. (1988) Inter- and intra-locus linkage analysis in Sordaria fimicola. Fungal Genet. Newsletter 35, 16-21.

KITANI, Y. and OLIVE, L. S. (1967) Genetics of Sordaria fimicola VI. Gene conversion at the $g$ locus in mutant $\mathrm{x}$ wild type crosses. Genetics 57, 767-782.

KITANI, Y. and OLIVE, L. S. (1969) Genetics of Sordaria fimicola. VII. Gene conversion at the $g$ locus in interallelic crosses. Genetics 62, 23-66.

KITANI, Y. and OLIVE, L. S. (1970) Alteration of gene conversion paterns in Sordaria fimicola by supplementation with DNA bases. Proc. Natl. Acad. Sci. USA 66, 1290-1297.

KitANI, Y. and WhITEhouse, H. L. K. (1974) Effect of the proportion of parental nuclei in a heterokaryon on the patern of gene conversion in Sordaria fimicola. Mol. Gen. Genet. 131, 47-56.

KLEIN, H. L. (1984) Lack of association between intrachromosomal gene conversion and reciprocal exchange. Nature 310, 748-753.

LACADENA, J. R. and FerRer, E. (1978) Homologous somatic association in Crepis species: A critical comparison of several statistical apperoaches. Chromosoma 67, 77-86.

LANB, B. C. and Wickramaratne, M. R. T. (1973) Corresponding-site inter-ference, synaptonemal complex structure, and 8+: On and 7+1m octads from wild type x mutant crosses of Ascobolus immersus. Genet. Res. (Camb.) 22, 113-124.

MaguIR, M. P. (1983) Chromosome behavior at premeiotic mitosis in maize. J. Heredity 74, 93-96.

Meselson, M. S. and Radding, C. M. (1975) A general model for genetic recombination. Proc. Natl. Acad. Sci. USA 72, 358-361.

PAQUeTTE, N. (1978) Detection of aberrant 4:4 asci in Ascobolus immersus. Canad. J. Genet. Cytol. 20, 9-17. 
Perkins, D. D., EL-ANI, A. S., OLive, S. and Kitani, Y. (1936) Interference between exchanges in tetrads of Sordaria fimicola. Am. Naturalist 97, 249-252.

PONTECORvo, G. and CÁFER, E. (1958) Genetic analysis based on mitotic recombination. Adv: Genet. 9, 71-104.

Pontecorvo, G., Roper, J. A., Hemmons, L. M., MacDnald, and Bufton, A. W. J. (1953) The genetics of Aspergillus nidulans. Adv. Genet. 5, 141-238.

PRITChARD, R. H. (1960) Localized negative interference and its bearing on models of gene recombination. Genet. Res. (Camb.) 1, 1-24.

Putrament, A. (1967) On the mechanism of mitotic recombination in Aspergillus nidulans. I. Intragenic recombination and DNA replication. Mol. Gen. Genet. 100, 307-320.

Putrament, A. (1967) On the mechanism of mitotic recombination in Aspergillius nidulans. II. Simultaneous recombination within two closely linked cistrons. Mol. Gen. Genet. 100, $321-336$.

RAo, R. M. and NATARAJAN, A. T. (1967) Somatic association in relation to chemically induced chromosome aberrations in Vicia faba. Genetics 57, 821-835.

Rieger, R., Michaelis, A., Schubert, I. and Meister, A. (1973) Somatic interphase pairing of Vicia chromosomes as inferred from the hom/het ratio of induced chromatid interchanges. Mutation Res. 20, 295-298.

Roman, H. and FABRE, F. (1983) Gene conversion and associated reciprocal recombination are separable events in vegetative cells of Saccharo-myces cerevisiae. Proc. Natl. Acad. Sci. USA 80, $6912-6916$.

Singh, R. J., RöBbELEN, G. and OKAMOTo, M. (1976) Somatic association at interphase studied by Giemsa banding Technique. Chromosoma 56, 265-273.

STACK, S. M. and ClARKE, C. R. (1973) Differential Giemsa-staining of the telomeres of Allium cepa chromosomes: observations related to chromosome pairing. Canad. J. Genet. Cytol. 15, 619-624.

STERN, C. (1936) Somatic crossing over and segregation in Drosophila melanogaster. Genetics 21, 625-730.

StERn, C. (1969) Somatic recombination within the white locus of Droso-phila melanogeaster. genetics 62, 573-581.

Svichenko, S. I. (1975) Somatic associations of chromosomes. Taitol. Genet. 9, 464-470.

van HEEMERT, C. (1977) Somatic pairing and meiotic nonrandom disjunction in a pericentric inversion of Hylemya antiqua (Heigen). Chromosoma 59, 193-206.

VIG, K. (1973) Somatic crossing over in Glycine max (L.) Merrill: Effect of some inhibitors of DNA synthesis in the induction of somatic crossing over and point mutations. Genetics 73, 583-596.

WAGENAAR, E. B. (1969) End-to-end chromosome attachments in mitotic interphase and their possible significance to mitotic chromosome pairing. Chromosoma 26, 410-426.

WILLIS, K. K: and KLEIN, H. L. (1987) Intrachromosomal recombination in Saccharomyces cerevisiae: reciprocal exchange in an inverted repeat and associated gene conversion. Genetics 117, 633-643.

YOSHIDA, H. and YAMAGUCHI, H. (1973) Arrangement and association of somatic chromosomes induced by chloramphenicol in barley. Chromosoma 43, 399-407. 